\title{
Surface Functionalization of Graphene Oxide with Polymer Brushes for Improving Thermal Properties of the Polymer Matrix
}

\author{
Shuangshuang Wang $\left(\mathbb{D},{ }^{1}\right.$ Houfang Chi $\mathbb{D},{ }^{1}$ Lin Chen $\mathbb{D}^{1},{ }^{1}$ Wei Li $\mathbb{D}^{2},{ }^{2}$ Yuchao Li $\mathbb{D}$, \\ Guang Li $\mathbb{D}^{1}$, and Xiangcai Ge $\mathbb{D}^{1}$ \\ ${ }^{1}$ School of Materials Science and Engineering, Liaocheng University, Liaocheng 252059, China \\ ${ }^{2}$ Key Laboratory of Rubber-Plastics, Ministry of Education/Shandong Provincial Key Laboratory of Rubber-Plastics, \\ Qingdao University of Science \& Technology, Qingdao 266042, China
}

Correspondence should be addressed to Shuangshuang Wang; wangshuangshuang@lcu.edu.cn

Received 5 February 2021; Revised 2 April 2021; Accepted 6 May 2021; Published 15 May 2021

Academic Editor: Gyorgy Szekely

Copyright (c) 2021 Shuangshuang Wang et al. This is an open access article distributed under the Creative Commons Attribution License, which permits unrestricted use, distribution, and reproduction in any medium, provided the original work is properly cited.

\begin{abstract}
In this work, polymethyl methacrylate (PMMA) and polystyrene (PS) with controlled structures would be grafted on graphene material. The hybrid materials were prepared by coating graphene oxide (GO) with polydopamine (PDA) as a reactive underlayer and reducing agent, subsequently, surface-initiated polymerization of monomers (methyl methacrylate, styrene) based on the activators regenerated electron transfer atom transfer radical polymerization (ARGET-ATRP) technique. The polymer brush-modified graphene materials were then incorporated into the PMMA or PS matrix to get polymer nanocomposites with better thermal properties. The results of Fourier transform infrared spectroscopy (FTIR), X-ray photoelectron spectroscopy (XPS), scanning electron microscopy (SEM), transmission electron microscopy (TEM), and thermal gravimetric analysis (TGA) demonstrated that PMMA and PS chains were successfully anchored on the surfaces of functionalized GO sheets. The influence of the grafted polymer brush-modified GO on thermal stability of PMMA and PS was investigated by a simultaneous thermal analyzer. Thermal conductivity of the polymer nanocomposite was determined by a conductive calorimeter. The results showed that thermal stability, glass transition temperature $\left(T_{\mathrm{g}}\right)$, and thermal conductivity of the polymer nanocomposites were obviously improved compared with pure PMMA or PS.
\end{abstract}

\section{Introduction}

Graphene has emerged to be a promising material due to its unique two-dimensional structure and a range of prominent properties. Graphene-based materials have attracted considerable attention because of their excellent mechanical, electrical, optical, and thermal properties [1-4]. In recent several years, more attention has been drawn to use graphene as a potential reinforcement filler in the generation of polymer composites with the aim of increasing the properties of the polymer composites, such as thermal conductivity, mechanical, and adsorption properties [5-8]. However, graphene sheets tend to agglomerate through van der Waals interac- tions due to the high specific surface area, which greatly reduced the final material properties. Preparation of welldispersible graphene-polymer composites is an urgent need in both academia and industry. Up to now, graphene oxide (GO) has been considered as an excellent precursor to various graphene-based materials due to its large-scale and cost-effective preparation. A number of methods have been explored to disperse graphene into polymer matrices, including covalent and noncovalent functionalization [9-13]. The dispersity of graphene nanosheets could be facilitated by noncovalent functionalization, such as $\pi-\pi$, van der Waals, and electrostatic interactions between polymeric materials and graphene [14-16]. Bioinspired polydopamine chemistry 


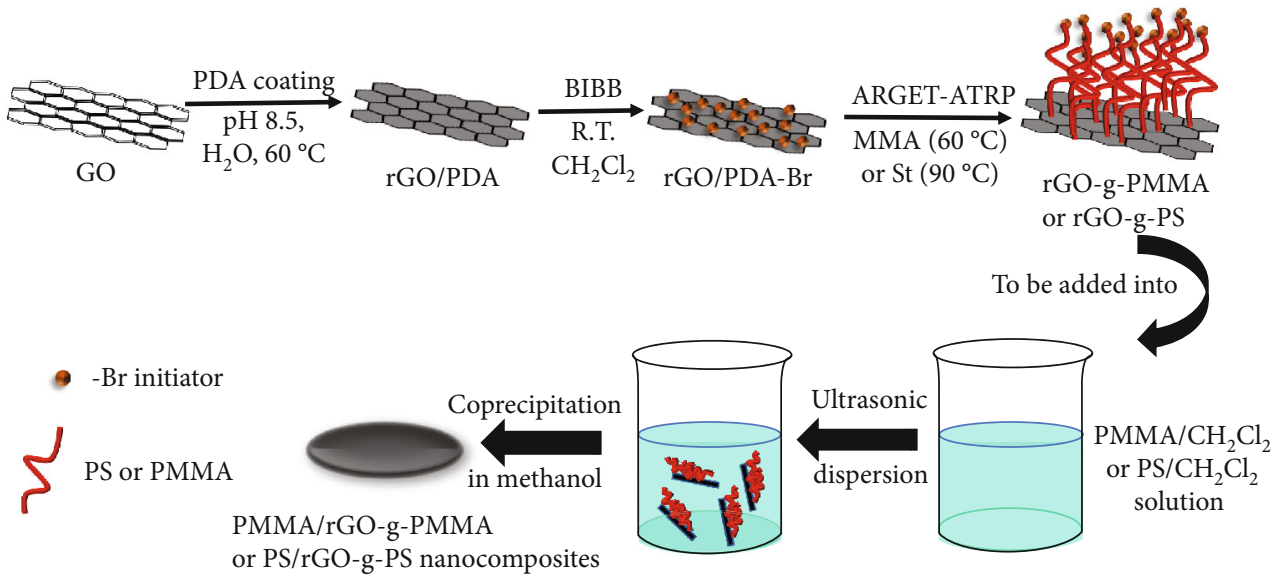

Figure 1: The schematic for preparation of rGO-g-PMMA, rGO-g-PS, PMMA/rGO-g-PMMA, and PS/rGO-g-PS.

is an efficient and universal surface noncovalent functionalization strategy in recent years, owing to the strong adhesion of polydopamine (PDA) and the high density of reactive groups on the PDA coating [17]. Additionally, dopamine (DA) has been proved to be an environment-friendly reducing agent for GO [18-21]. Covalent functionalization of graphene with polymer brushes via either "grafting from" or "grafting to" approaches is also an effective strategy for surface modification [22-24]. Compared with the "grafting to" method, the "grafting from" approach shows the advantages of creating densely and well-controlled grafted polymer brushes. Until now, graphene modified with various polymers has been synthesized by using the "grafting from" approach, which generally based on controlled living radical polymerization, such as atom transfer radical polymerization (ATRP), reversible addition-fragmentation chain transfer polymerization (RAFT), single electron transfer-living radical polymerization (SET-LRP), nitroxide-mediated polymerization (NMP), and photo-induced copper-mediated polymerization (SI-photo CMP) [25-38]. In comparison with ATRP, ARGET-ATRP is a potentially more industrially attractive methodology, which minimizes the copper catalyst to ppm levels due to the existing excess reducing agent. Moreover, ARGET-ATRP also has good atmosphere tolerance. Although many different polymers have been grafted from various solid substrates via the ARGET-ATRP technique [39-43], grafting polymer brushes from graphene materials by ARGET-ATRP is still scarcely reported $[44,45]$.

Grafting of polymers from solid substrates by the combination of PDA chemistry and ARGET-ATRP has been firstly reported in our previous work $[45,46]$. Preparation of grafted polymer-functionalized reduced graphene oxide ( $\mathrm{rGO}$ ) by PDA chemistry and ARGET-ATRP has not been reported by other research groups. Herein, PMMA and PS were grafted from functionalized GO, which was prepared by PDA noncovalent modification combined with ARGET-ATRP covalent modification. As shown in Figure 1, GO was first coated with a homogeneous bioadhesive PDA layer via a simple in situ self-polymerization of $\mathrm{DA}$, and the product $\mathrm{rGO} / \mathrm{PDA}$ was obtained. Then, the initiator, 2-bromoisobutyryl bromide

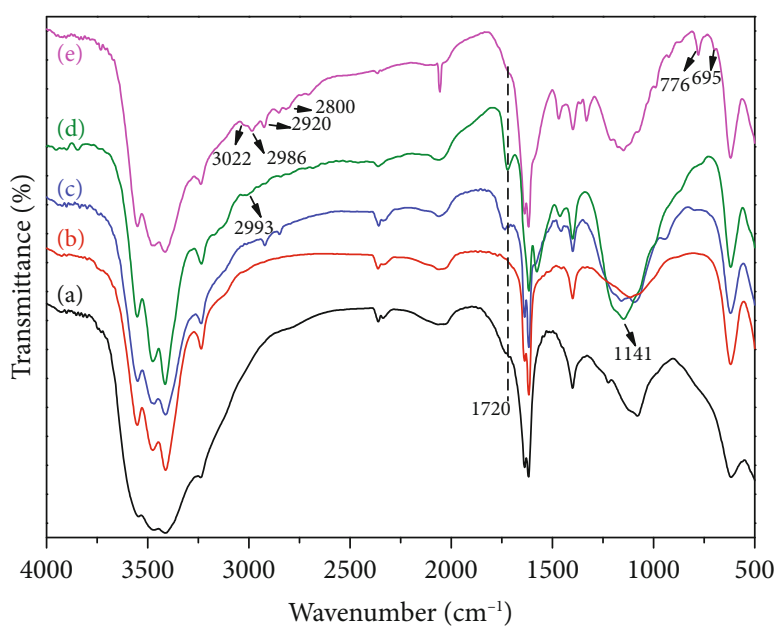

FIgURE 2: FTIR spectra of GO (a), rGO/PDA (b), rGO/PDA-Br (c), rGO-g-PMMA (d), and rGO-g-PS (e).

(BIBB), was coupled onto the surface of rGO/PDA nanosheets to initiate the polymerization of MMA and St by using the ARGET-ATRP technique. The grafted PMMA- and PSfunctionalized rGO were named rGO-g-PMMA and rGO-gPS, respectively. Finally, PMMA- and PS-functionalized rGO were used as nanofillers in the PMMA and PS matrices, respectively, via solution compounding to obtain PMMA/rGO-gPMMA or PS/rGO-g-PS nanocomposites.

Benefiting from the grafted polymer chains, rGO-gpolymer nanosheets exhibited good compatibility with the pure polymer matrix. The grafted polymer-functionalized rGO played an important role in the enhancement of thermal stability and thermal conductivity of the prepared polymer nanocomposites. In this work, the applied method combined both the advantages of noncovalent and covalent methods. We presented a general, convenient, and green methodology for preparing polymer brush-functionalized $\mathrm{rGO}$, and the polymer-modified rGO could be used as a nanofiller to improve the thermal properties of the polymer matrix. 


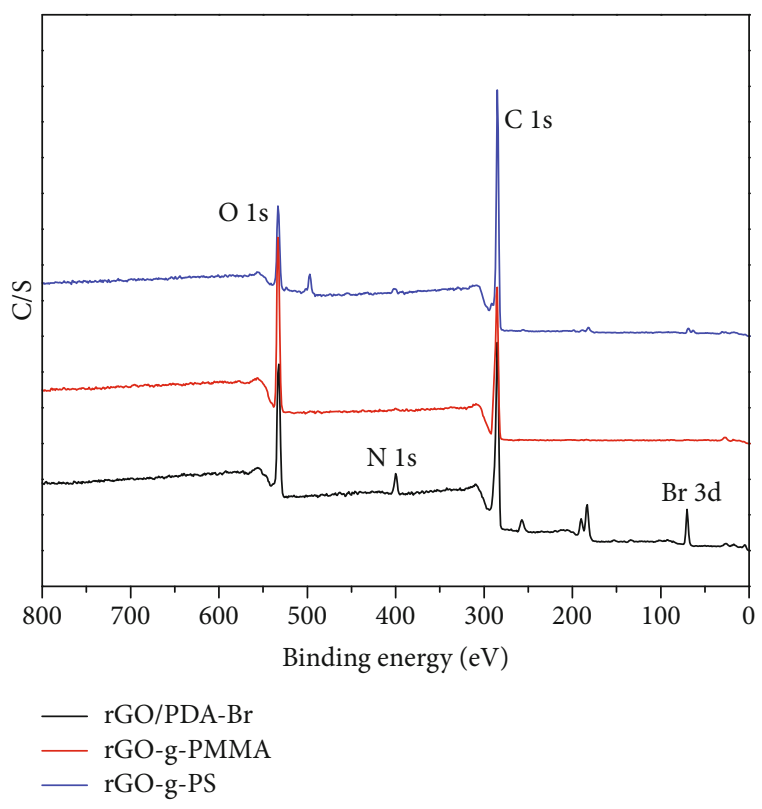

(a)

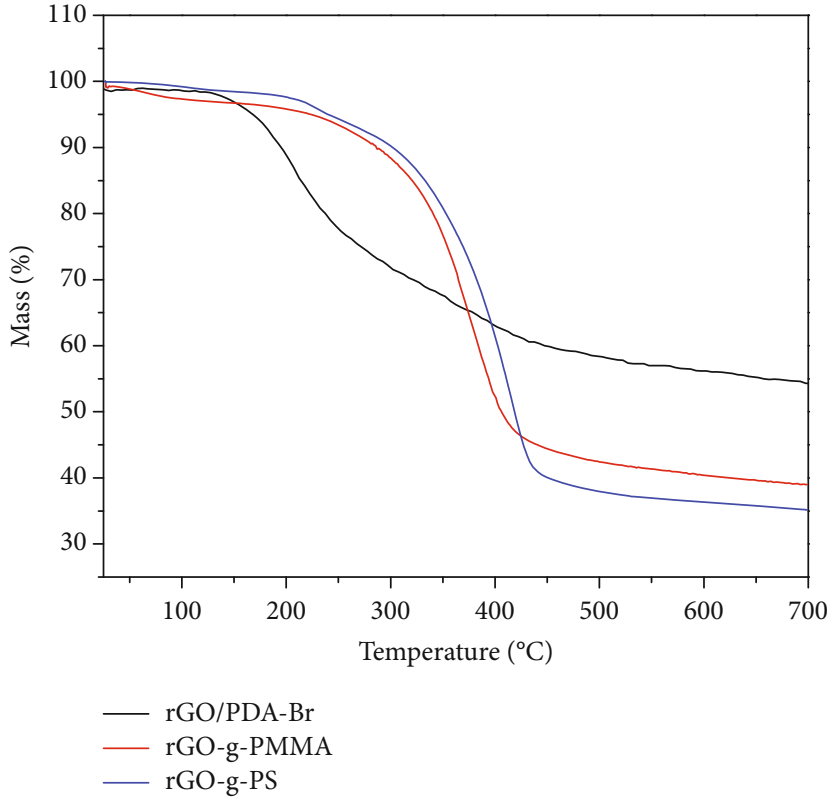

(b)

Figure 3: XPS spectra (a) and TGA curves (b) of rGO-g-PMMA and rGO-g-PS.

\section{Experimental}

2.1. Materials. Graphene oxide was purchased from Yuanye Biological Technology Co., Ltd. (Shanghai, China). Tris base (99\%), copper(II) bromide $\left(\mathrm{CuBr}_{2}, 99 \%\right)$, dopamine hydrochloride (DA, 99\%), ascorbic acid (AsAc, 99\%), methyl methacrylate (MMA), and styrene (St) were purchased from Aladdin Reagents Co., Ltd. (Shanghai, China). MMA and St were purified by passing through a column filled with basic alumina to remove inhibitors before use. 2-Bromoisobutyryl bromide (BIBB, 98\%), $N, N, N^{\prime}, N{ }^{\prime \prime}, N{ }^{\prime \prime}$-pentamethyldiethylene triamine (PMDETA, 99\%), and ethyl 2-bromoisobutyrate (EBIB) were purchased from TCI Development Co., Ltd. (Shanghai, China) and used as received. Other analytical grade chemicals were commercially obtained and used directly.

2.2. Characterization. Fourier transform infrared spectroscopy (FTIR) spectra (in $\mathrm{KBr}$ pellets) were recorded on a Nicolet-100 spectrometer in the range of $400-4000 \mathrm{~cm}^{-1}$, and the measurement data was collected in transmittance mode. Surface elemental composition of the grafted PMMAand PS-functionalized rGO was determined by X-ray photoelectron spectroscopy (XPS). All XPS spectra were recorded on an ESCALAB $250 \mathrm{Xi}$ spectrometer (Thermo Fisher Scientific, USA) with a microfocused monochromated $\mathrm{Al} \mathrm{K} \alpha \mathrm{X}$ ray source. SEM images of PMMA- and PS-functionalized graphene were obtained on a field emission scanning electron microscopy (FESEM) instrument (Zeiss Merlin Compact, Germany) with an accelerating voltage of $3 \mathrm{kV}$. Microstructures of grafted polymer-functionalized rGO were observed by transmission electron microscopy (TEM), which were carried out on an FEI Tecnai F30 TEM (USA). Size exclusion chromatography (SEC) was performed to determine the molecular weight and polydispersity of the grafted polymer by using a Waters 1515 isocratic HPLC pump and Waters 2414 RI detector. THF was used as eluent with a flow rate of $1.0 \mathrm{~mL} / \mathrm{min}$ at $35^{\circ} \mathrm{C}$, and narrow molecular weight distribution (MWD) linear polystyrene standards were used for the calibration. Glass transition temperature $\left(T_{\mathrm{g}}\right)$ and thermal gravimetric analysis curves were investigated by a NETZSCH Simultaneous Thermal Analyzer (STA449, Germany). The samples were scanned in the temperature range of $30^{\circ} \mathrm{C}-800^{\circ} \mathrm{C}$ at a heating rate of $10^{\circ} \mathrm{C} / \mathrm{min}$ under a nitrogen atmosphere $(20 \mathrm{~mL} / \mathrm{min})$. Thermal conductivity of PMMA/rGO-g-PMMA and PS/rGO-g-PS was measured by a DTC-300 conductive calorimeter (TA Instruments, USA). The diameter of the sample was $50 \mathrm{~mm}$, and the measurements were carried out at about $25^{\circ} \mathrm{C}, 40^{\circ} \mathrm{C}, 60^{\circ} \mathrm{C}$, and $80^{\circ} \mathrm{C}$, respectively.

2.3. Preparation of $r G O / P D A$ and $r G O / P D A-B r$. The preparation procedures of polydopamine-coated reduced graphene oxide (rGO/PDA) and BIBB-coated rGO/PDA (rGO/PDA-Br) were the same as our previous report [45]. The general preparation processes were as follows.

$200 \mathrm{mg}$ of GO and $100 \mathrm{mg}$ of dopamine hydrochloride were added into $400 \mathrm{~mL}$ of $50 \mathrm{mM}$ Tris- $\mathrm{HCl}$ solution $(\mathrm{pH}=8.5)$ and dispersed by sonication for $10 \mathrm{~min}$ in an ice bath. The mixture was vigorously stirred at $60^{\circ} \mathrm{C}$ for $24 \mathrm{~h}$, and then $\mathrm{rGO} / \mathrm{PDA}$ was obtained via repeated washing and centrifugation with water, acetone, and dichloromethane until the supernatant was clear (yield: $190 \mathrm{mg}$ ).

About $180 \mathrm{mg}$ of $\mathrm{rGO} / \mathrm{PDA}$ was dispersed in dried trichloromethane $(23.6 \mathrm{~mL})$, triethylamine $(2.25 \mathrm{~mL})$, and DMAP $(4.03 \mathrm{mmol})$ by sonication for $10 \mathrm{~min}$ in an ice bath. After that, BIBB (2 mL) was added dropwise to the reaction 

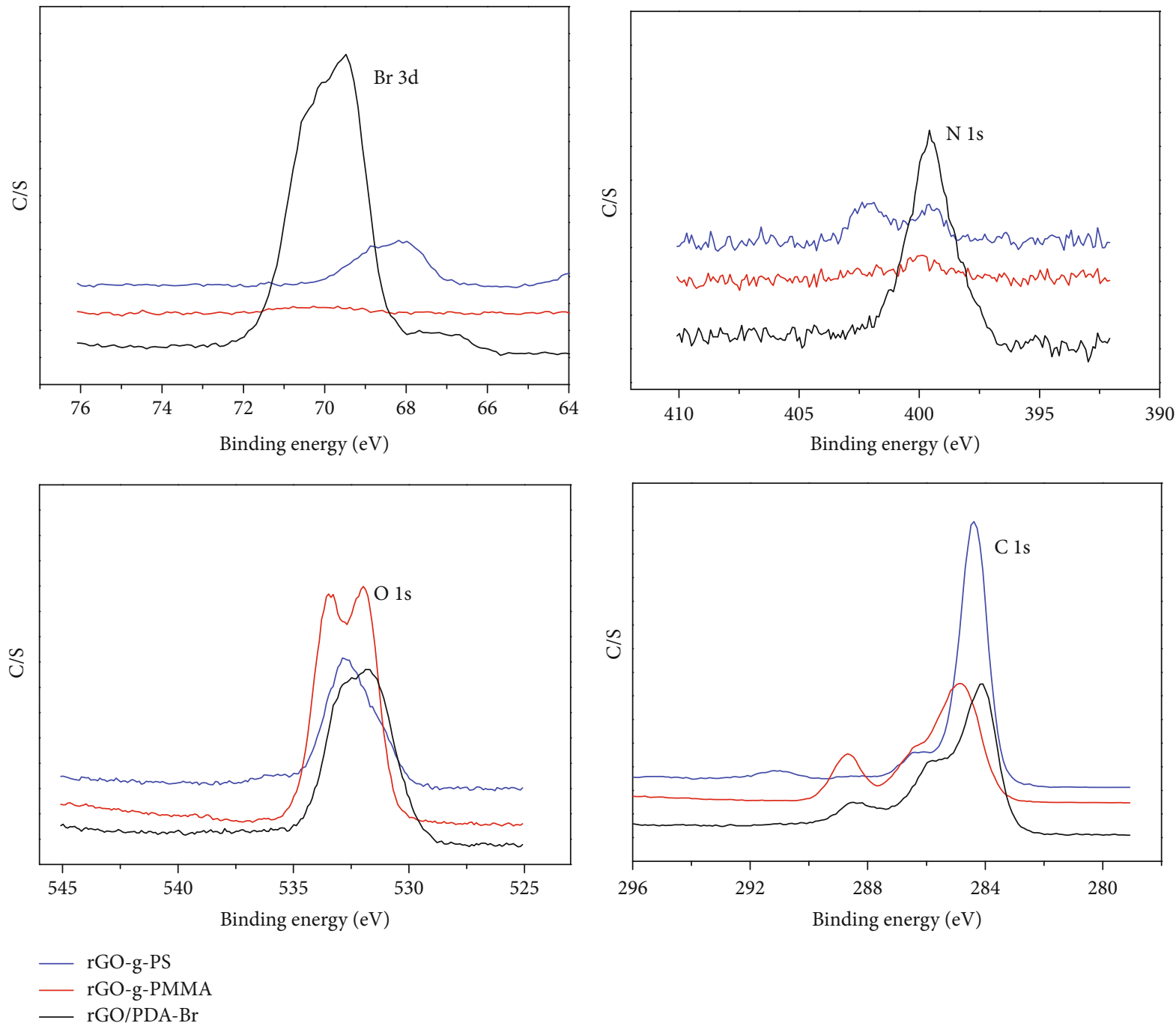

Figure 4: High-resolution scans of $\mathrm{C}$ 1s, O 1s, N 1s, and Br 3d of rGO-g-PMMA and rGO-g-PS.

mixture at $0^{\circ} \mathrm{C}$. The reaction was allowed to warm to room temperature and stirred overnight under a nitrogen atmosphere. $\mathrm{rGO} / \mathrm{PDA}-\mathrm{Br}$ was obtained via repeated washing and centrifugation with water, acetone, and dichloromethane. The samples were finally dried at $45^{\circ} \mathrm{C}$ under reduced pressure (yield: $170 \mathrm{mg}$ ).

2.4. Preparation of $r G O-g-P M M A$ and $r G O-g-P S$. rGO/PDA$\mathrm{Br}(20 \mathrm{mg})$ was dispersed in dimethylformamide (DMF, $6 \mathrm{~mL}$ ) by sonication for $10 \mathrm{~min}$ in a $25 \mathrm{~mL}$ dried Schlenk flask. Then, MMA monomer $(4 \mathrm{~mL}, 0.038 \mathrm{~mol})$, EBIB $(14 \mu \mathrm{L}, 0.097 \mathrm{mmol}), \mathrm{CuBr}_{2}(0.0094 \mathrm{mmol})$, and PMDETA $(0.094 \mathrm{mmol})$ were added. The Schlenk flask was simply degassed and refilled with nitrogen three times, and ascorbic acid (AsAc, $16.6 \mathrm{mg}, 0.094 \mathrm{mmol}$ ) was quickly added. The polymerization procedure was carried out at $60^{\circ} \mathrm{C}$ with different reaction times. When the reaction was finished, the polymerization was stopped by exposing the reaction mixture to air, and the mixture was diluted with acetone. The obtained rGO-g-PMMA was separated by centrifugation and washed with water/acetone and dichloromethane several times. The
TABLE 1: Surface element composition of rGO-g-PMMA and rGOg-PS based on the XPS analysis.

\begin{tabular}{lcccc}
\hline \multirow{2}{*}{ Samples } & \multicolumn{4}{c}{ Atomic concentration } \\
& C 1s (\%) & N 1s (\%) & O 1s (\%) & Br 3d (\%) \\
\hline rGO/PDA-Br & 72.51 & 4.24 & 19.38 & 3.87 \\
rGO-g-PMMA & 75.17 & 1.74 & 22.83 & 0.26 \\
rGO-g-PS & 83.29 & 1.65 & 14.4 & 0.66 \\
\hline
\end{tabular}

product was dried under a vacuum (yield: $26.1 \mathrm{mg}$ ). The collected supernatant was concentrated and precipitated in excess of methanol to obtain the isolated free PMMA. The final products should be dried in a vacuum at $45^{\circ} \mathrm{C}$ for $48 \mathrm{~h}$. Preparation procedures of $\mathrm{rGO}-\mathrm{g}$-PS and free PS were similar to that of rGO-g-PMMA and isolated free PMMA. The molar ratio of $[\mathrm{St}]:[\mathrm{EBIB}]:\left[\mathrm{CuBr}_{2}\right]:[\mathrm{PMDETA}]:[\mathrm{AsAc}]$ is $500: 1: 0.1: 1: 1$, and the polymerization was carried out at $90^{\circ} \mathrm{C}$ (yield: $34.1 \mathrm{mg}$ ). 


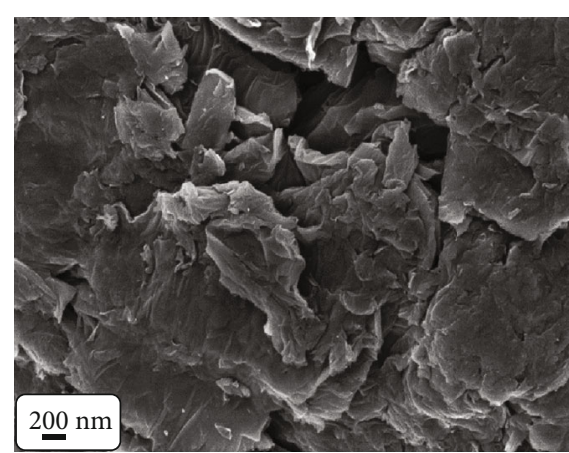

(a)

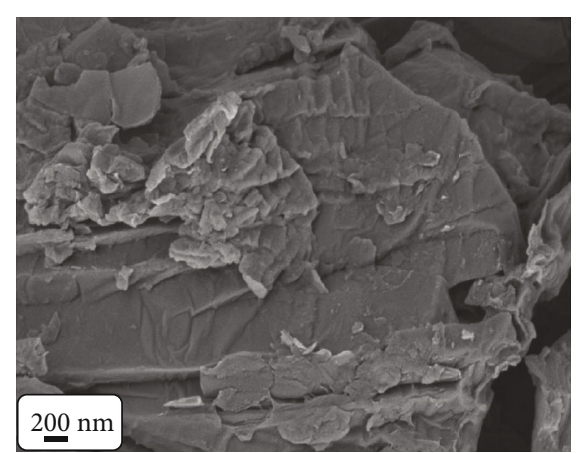

(b)

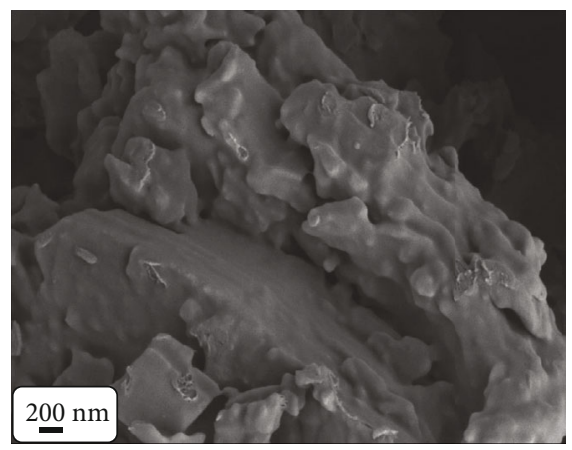

(c)

Figure 5: SEM images of GO (a), rGO-g-PMMA (b), and rGO-g-PS (c).

2.5. Preparation of PMMA/rGO-g-PMMA and PS/rGO-g-PS Nanocomposites. The collected free PMMA (reaction time: $4 \mathrm{~h}$ ) and PS (reaction time: $5 \mathrm{~h}$ ) would be used as the polymer matrix for PMMA/rGO-g-PMMA or PS/rGO-g-PS nanocomposites. rGO-g-PMMA (0.13g) and isolated free PMMA $(1.53 \mathrm{~g})$ were dispersed in dichloromethane $(40 \mathrm{~mL})$ by ultrasonication for $10 \mathrm{~min}$. Then, the mixture was coprecipitated in excess of methanol to obtain PMMA/rGO-g-PMMA composite; the final product was thoroughly dried in a vacuum at $45^{\circ} \mathrm{C}$ before use. The preparation procedure of PS/rGO-g-PS was the same as that of PMMA/rGO-g-PMMA.

\section{Results and Discussion}

3.1. Structure and Morphology of Grafted PolymerFunctionalized rGO. FTIR spectra of the grafted PMMA- or PS-functionalized rGO materials are shown in Figure 2. The FTIR spectra of GO, rGO/PDA, and rGO/PDA-Br have been discussed in our previous report [45]. In the FTIR spectrum of rGO-g-PMMA, a sharp absorbance peak at $1720 \mathrm{~cm}^{-}$ ${ }^{1}$ of the $\mathrm{C}=\mathrm{O}$ characteristic band of ester groups introduced by grafted PMMA was observed. Absorbances at about $2993 \mathrm{~cm}^{-1}$ and $1141 \mathrm{~cm}^{-1}$ were due to $\mathrm{C}-\mathrm{H}$ asymmetric stretching of methylene groups and $\mathrm{C}-\mathrm{O}$ stretching vibration. For rGO-g-PS, the FTIR spectrum showed bands assigned to the aromatic $\mathrm{C}-\mathrm{H}$ bonds $\left(3022 \mathrm{~cm}^{-1}\right)$ and methylene groups of styrene units $\left(2986-2800 \mathrm{~cm}^{-1}\right)$. Additionally, peaks at 776 and $695 \mathrm{~cm}^{-1}$ were attributed to the $\mathrm{C}-\mathrm{H}$ outof-plane ring bending and $\mathrm{C}-\mathrm{C}$ out-of-plane ring deforma- tion band, respectively. The FTIR spectra clearly confirmed that PMMA and PS chains have been anchored on the surface of graphene materials via the ARGET-ATRP procedure.

Surface element composition change after polymer grafting was examined by XPS. For rGO-g-PMMA and rGO-gPS, both the $\mathrm{N} 1 \mathrm{~s}(399.5 \mathrm{eV})$ and $\mathrm{Br} 3 \mathrm{~d}(69.5 \mathrm{eV})$ signals nearly disappeared (Figures 3(a) and 4). Moreover, the intensity of $\mathrm{O}$ 1s peaks for rGO-g-PMMA at $531.9 \mathrm{eV}$ and $533.3 \mathrm{eV}$ assigned to $\mathrm{C}=\mathrm{O}$ and $\mathrm{C}-\mathrm{O}$ was obviously increased in comparison with that for $\mathrm{rGO} / \mathrm{PDA}-\mathrm{Br}$ (Figure 4, O 1s). The successful grafting of PS brushes from rGO surfaces could also be confirmed by the obvious intensity decrease of the $\mathrm{O} 1 \mathrm{~s}$ signal $(532.8 \mathrm{eV})$ and intensity increase of the $\mathrm{C}$ 1s signal $(284.4 \mathrm{eV})$ (Figure $4, \mathrm{O} 1 \mathrm{~s}$ and $\mathrm{C} 1 \mathrm{~s})$. The percentages of $\mathrm{C} 1 \mathrm{~s}, \mathrm{~N} 1 \mathrm{~s}, \mathrm{O} 1 \mathrm{~s}$, and $\mathrm{Br} 3 \mathrm{~d}$ in grafted polymerfunctionalized $\mathrm{rGO}$ determined by XPS are given in Table 1. Element percentages of GO, rGO/PDA, and rGO/PDA-Br had been discussed in our previous work [45]. The variation trend of element percentages for $\mathrm{C} 1 \mathrm{~s}, \mathrm{~N}$ 1s, $\mathrm{O} 1 \mathrm{~s}$, and $\mathrm{Br} 3 \mathrm{~d}$ in rGO-g-PMMA and rGO-g-PS was consistent with the results of XPS spectra. For rGO-g-PMMA, the element percentage of $\mathrm{N}$ 1s and $\mathrm{Br} 3 \mathrm{~d}$ was decreased to $1.74 \%$ and $0.26 \%$, respectively; however, the $\mathrm{O} 1 \mathrm{~s}$ ratio was increased to $22.83 \%$. The element percentage of $\mathrm{N} 1 \mathrm{~s}$ and $\mathrm{Br} 3 \mathrm{~d}$ was decreased to $1.65 \%$ and $0.66 \%$ for rGO-g-PS, and the C 1 s ratio was increased to $83.29 \%$.

The percentage of PMMA or PS grafted on rGO could be determined by TGA, and the results are given in Figure 3(b). It could be observed that both rGO-g-PMMA and rGO-g-PS 


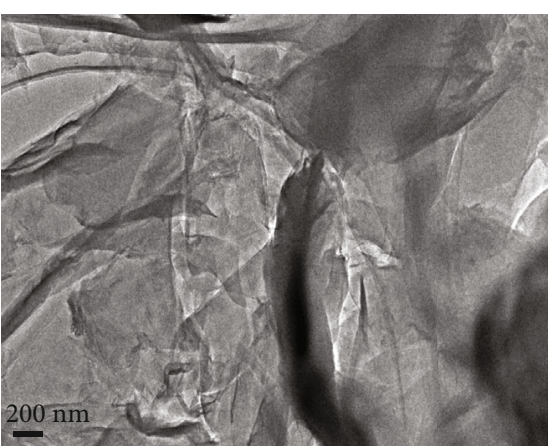

(a)

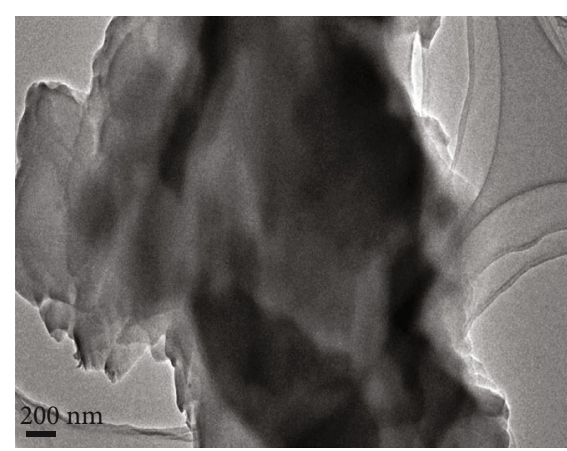

(b)

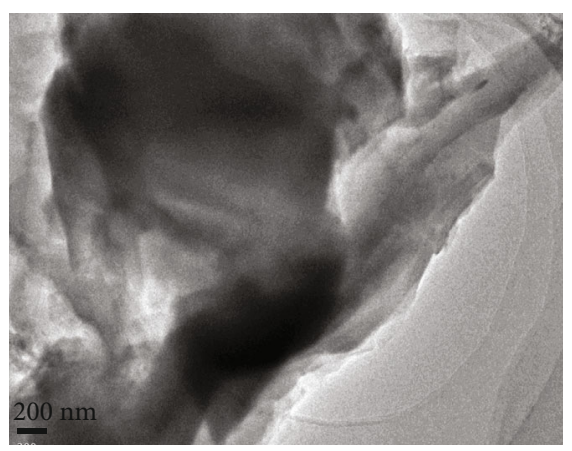

(c)

Figure 6: TEM images of GO (a), rGO-g-PMMA (b), and rGO-g-PS (c).

exhibited sharp weight loss at the range of $300^{\circ} \mathrm{C}-450^{\circ} \mathrm{C}$, which was caused by the thermal decomposition of grafted polymer backbones. TGA curves of PMMA-functionalized rGO showed $61.15 \mathrm{wt} \%$ weight loss at $700^{\circ} \mathrm{C}$, indicating that the weight percentage of grafted PMMA was about 38.85 wt $\%$ for rGO-g-PMMA. For rGO-g-PS, it could be estimated that approximately $35.27 \mathrm{wt} \%$ of PS was grafted from the surface of rGO.

SEM observation was performed to detect morphological changes after PMMA and PS brushes were grafted on functionalized rGO, and SEM images are given in Figure 5. Surface morphologies of GO, rGO/PDA, and rGO/PDA-Br determined by SEM have been discussed previously [45]. As shown in Figure 5, surface morphologies of rGO-gPMMA and rGO-g-PS were different from that of GO. After polymer grafting, a rather coarse and dense surface would be observed in the surface of rGO-g-PMMA and rGO-g-PS, indicating that the functionalized GO had been covered with PMMA or PS chains.

The morphologies of the grafted PMMA- and PSmodified rGO samples were also studied by TEM, and the results are given in Figure 6. According to our previous publication [45], the GO sheet exhibited few-layer planar sheets. However, both images of rGO-g-PMMA and rGOg-PS exhibited thick plates with dark contrast after polymer grafting (images (b) and (c)), demonstrating that the surface of functionalized rGO sheets has been covered by PMMA and PS chains. Thus, TEM images clearly indicated the success of the formation of grafted polymer brushes from the rGO surface.
TABLE 2: $M_{\mathrm{n}}, M_{\mathrm{w}}$, and PDI values of isolated free PMMA with different polymerization times.

\begin{tabular}{lccc}
\hline Polymerization time $(\mathrm{h})$ & $M_{\mathrm{n}}(\mathrm{kDa})$ & $M_{\mathrm{w}}(\mathrm{kDa})$ & PDI \\
\hline 2 & 27.68 & 47.21 & 1.71 \\
4 & 38.29 & 67.27 & 1.76 \\
8 & 51.23 & 91.72 & 1.79 \\
\hline
\end{tabular}

TABLE 3: $M_{\mathrm{n}}, M_{\mathrm{w}}$, and PDI values of isolated free PS with different polymerization times.

\begin{tabular}{lccc}
\hline Polymerization time $(\mathrm{h})$ & $M_{\mathrm{n}}(\mathrm{kDa})$ & $M_{\mathrm{w}}(\mathrm{kDa})$ & PDI \\
\hline 3 & 10.38 & 21.40 & 2.06 \\
9 & 31.96 & 53.96 & 1.69 \\
11 & 41.97 & 72.50 & 1.73 \\
\hline
\end{tabular}

It has been reported that the free polymer initiated by the sacrificial initiator in polymerization solution has almost identical molecular weights to those formed from the solid matrix $[47,48]$. In order to demonstrate that the ARGETATRP technique was carried out in a controlled way, ethyl 2-bromoisobutyrate (EBIB) was used as the sacrificial initiator. The molecular weight $\left(M_{\mathrm{n}}, M_{\mathrm{w}}\right)$ and polydispersity index (PDI) of isolated free PMMA and PS formed from the sacrificial initiator were characterized by SEC. $M_{\mathrm{n}}, M_{\mathrm{w}}$, and PDI 


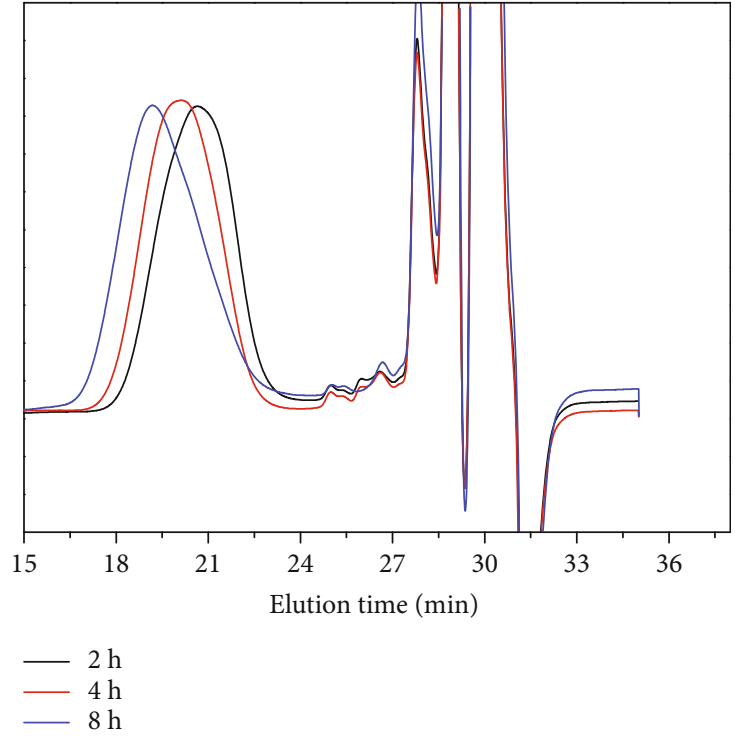

(a)

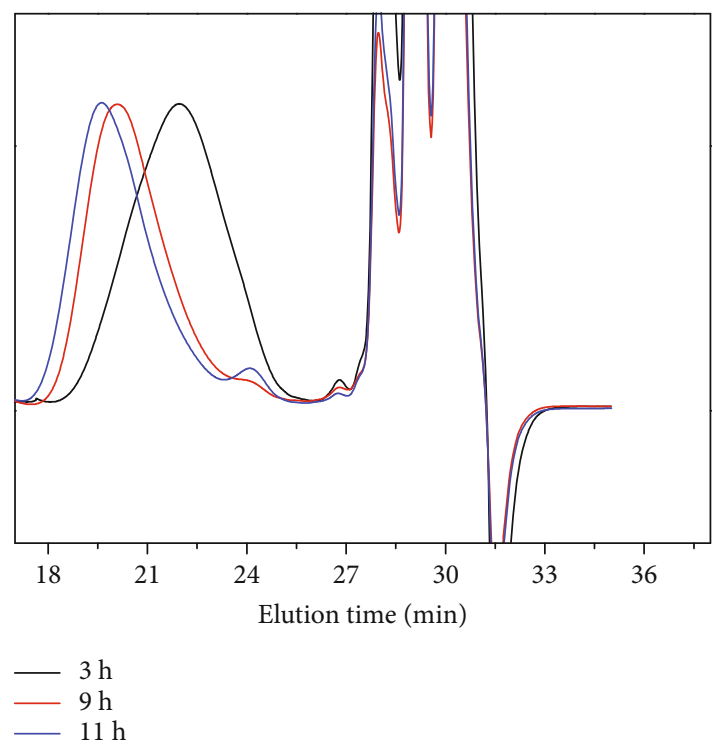

(b)

FIGURE 7: SEC traces for the series of isolated free polymethyl methacrylate (PMMA) (a) and polystyrene (PS) (b) with different polymerization times.
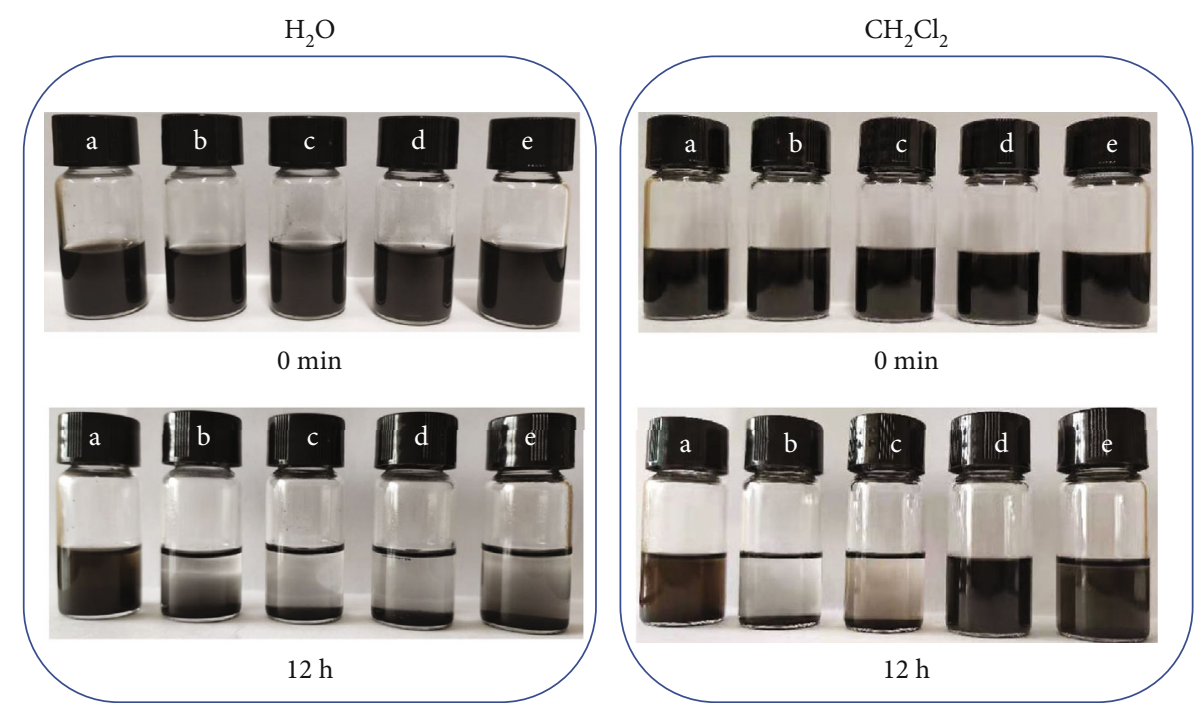

Figure 8: Dispersions of GO (a), rGO/PDA (b), rGO/PDA-Br (c), rGO-g-PS (d), and rGO-g-PMMA (e) in deionized water and dichloromethane immediately after sonication and $12 \mathrm{~h}$ after sonication (sonication time: $10 \mathrm{~min}$; concentration: $1 \mathrm{mg} / \mathrm{mL}$ ).

values of PMMA and PS are listed in Tables 2 and 3. Moreover, the obtained values could be used to characterize the surface-grafted PMMA or PS on rGO. As shown in Figure 7, both SEC curves of the free PMMA and PS exhibited monomodal distributions, and SEC traces shifted continuously toward higher molecular weight with increasing polymerization time, indicating the increased monomer conversion and thus enhanced polymerization degree of grafted polymers. These results suggested that ARGET-ATRP was carried out in a controlled way.

The dispersibility of PMMA- and PS-functionalized rGO sheets was investigated. GO, rGO-g-PMMA, and rGO-g-PS
TABLE 4: Glass transition temperature $\left(T_{\mathrm{g}}\right)$ values of PMMA/rGOg-PMMA and PS/rGO-g-PS nanocomposites.

\begin{tabular}{lc}
\hline Samples & $T_{\mathrm{g}}\left({ }^{\circ} \mathrm{C}\right)$ \\
\hline PMMA & 109.7 \\
PMMA/rGO-g-PMMA & 124.6 \\
PS & 92.8 \\
PS/rGO-g-PS & 105.8 \\
\hline
\end{tabular}




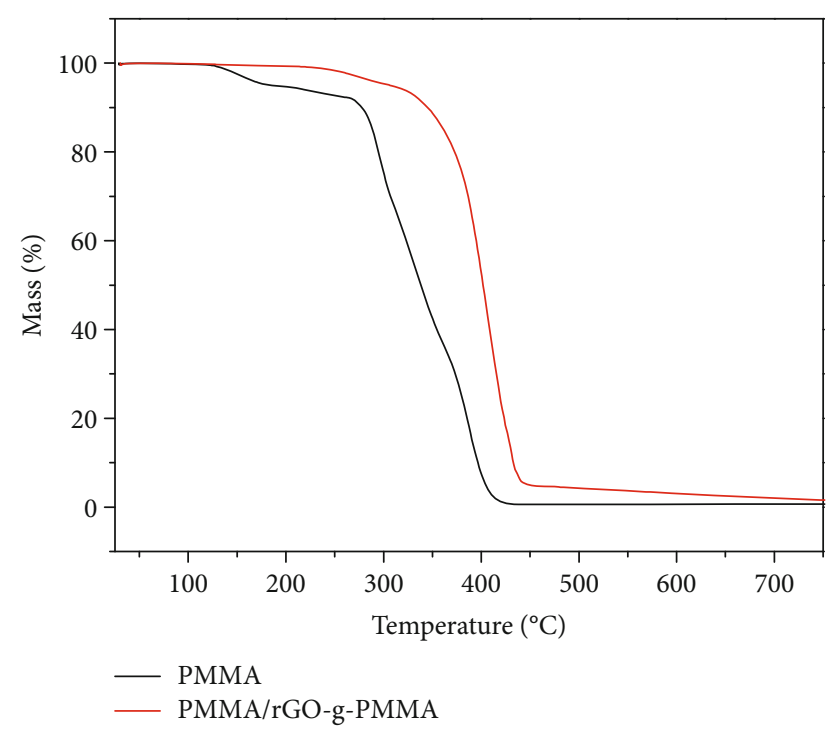

(a)

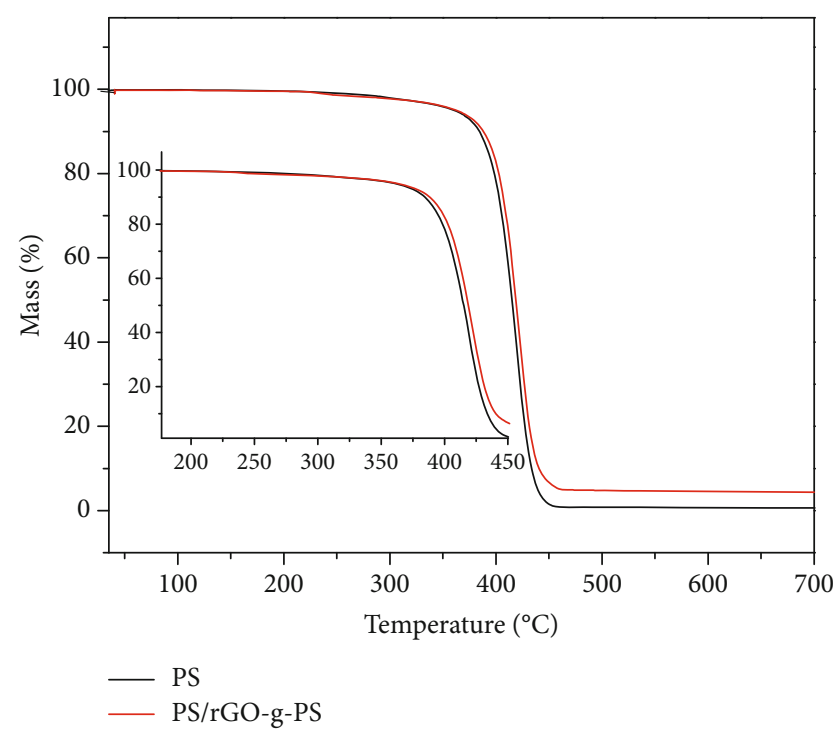

(b)

Figure 9: TGA curves of PMMA/rGO-g-PMMA, PS/rGO-g-PS, PMMA, and PS.

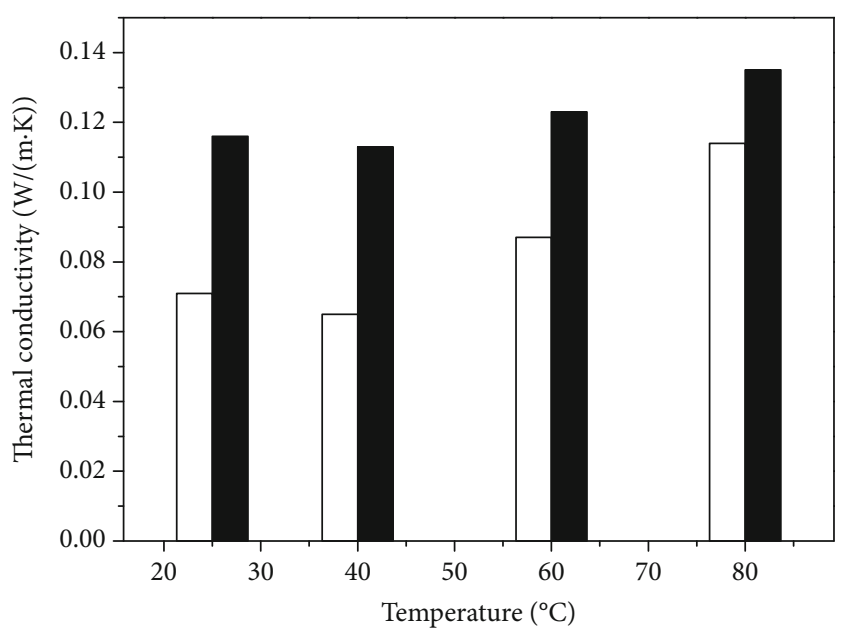

PMMA

PMMA/rGO-g-PMMA

(a)

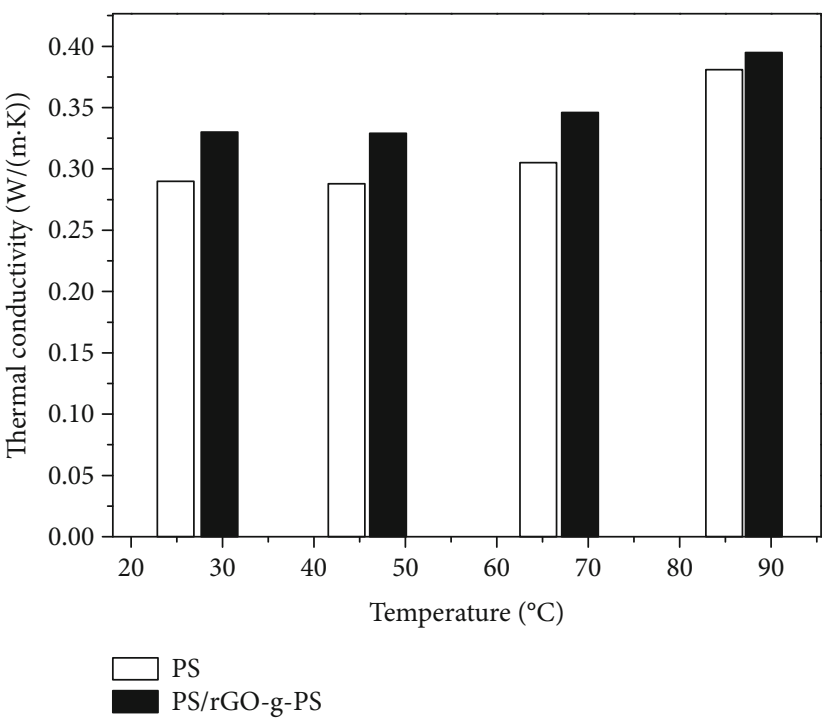

(b)

Figure 10: Thermal conductivity of PMMA/rGO-g-PMMA, PS/rGO-g-PS, PMMA, and PS.

were dispersed in deionized water $\left(\mathrm{H}_{2} \mathrm{O}\right)$ and dichloromethane $\left(\mathrm{CH}_{2} \mathrm{Cl}_{2}\right)$ via sonication $(10 \mathrm{~min}$, Figure 8$)$. After $12 \mathrm{~h}$, the dispersibility of rGO-g-PMMA and rGO-g-PS is poor in deionized water in comparison with that of GO, which could be attributed to the hydrophobic properties of the grafted polymer. However, rGO-g-PS and rGO-g-PMMA showed obviously enhanced dispersibility in organic solvent $\left(\mathrm{CH}_{2} \mathrm{Cl}_{2}\right)$ because of the well dissolving capacity of PS and PMMA in $\mathrm{CH}_{2} \mathrm{Cl}_{2}$. Even $12 \mathrm{~h}$ after sonication, the PS- and PMMA-modified rGO sheets did not obviously precipitate. The improved dispersibility of grafted polymer-modified rGO in organic solvent would promote its compatibility with the polymer matrix.
3.2. Thermal Stability of the Polymer/Functionalized $r G O$ Nanocomposites. The glass transition behavior of PMMA/rGOg-PMMA and PS/rGO-g-PS nanocomposites was explored, and the corresponding glass transition temperature $\left(T_{\mathrm{g}}\right)$ values are listed in Table 4 . When incorporated with $8 \mathrm{wt} \%$ of rGO-gPMMA, $T_{\mathrm{g}}$ of PMMA increased from 109.7 to $124.6^{\circ} \mathrm{C}$. $T_{\mathrm{g}}$ of PS increased from 92.8 to $105.8^{\circ} \mathrm{C}$ after the addition of $8 \mathrm{wt} \%$ rGO-g-PS in the PS matrix. The improvement of $T_{\mathrm{g}}$ values for polymer nanocomposites was attributed to the introduction of graphene nanosheets restricting the mobility of the polymer chains, which demonstrated the good interfacial interaction between functionalized graphene sheets and polymer chains. 
The influences of the functionalized rGO on the thermal stability of the polymer matrix were tested by TGA, as shown in Figure 9. The corresponding temperatures of $10 \%$ mass loss of PMMA and PMMA/rGO-g-PMMA were $279.3^{\circ} \mathrm{C}$ and $344.8^{\circ} \mathrm{C}$, respectively. The corresponding temperatures of 50\% mass loss of PMMA and PMMA nanocomposites were $339.2^{\circ} \mathrm{C}$ and $401.9^{\circ} \mathrm{C}$. Pure PS showed $10 \%$ mass loss and $50 \%$ mass loss at $383.7^{\circ} \mathrm{C}$ and $415.7^{\circ} \mathrm{C}$, respectively. After the addition of rGO-g-PS, the $10 \%$ mass loss and $50 \%$ mass loss of PS nanocomposites were increased to $388.8^{\circ} \mathrm{C}$ and $420.6^{\circ} \mathrm{C}$. The results indicated that $\mathrm{PMMA} / \mathrm{rGO}-\mathrm{g}-\mathrm{PMMA}$ and PS/rGO-g-PS nanocomposites have better thermal stability. The improvement in thermal stability of these polymer nanocomposites might be due to the high heat capacity and thermal conductivity of the dispersed graphene sheets. Besides, the interfacial interactions between the functionalized graphene and the polymer matrix can lead to the formation of physical or chemical crosslinking points which hinder the degradation of polymer chains $[49,50]$.

3.3. Thermal Conductivity. It is well known that the interfacial thermal resistance across a filler-matrix interface could limit the thermal conductivity of the composite [51]. Promoting interaction between the matrix and the filler could reduce phonon scattering at the matrix-filler interface, thus improving higher composite thermal conductivity [52]. Thermal conductivity results of PMMA/rGO-g-PMMA and PS/rGO-g-PS at different temperatures are given in Figure 10. The results showed that the addition of grafted polymer-functionalized rGO could obviously improve the interfacial heat transfer between the rGO and the polymer matrix. The thermal conductivity improvement of PMMA composites was about $66 \%$ higher than that of PMMA at $25^{\circ} \mathrm{C}$, and thermal conductivity improvement of PS composites was about $14 \%$ higher than that of PS at $25^{\circ} \mathrm{C}$. The good dispersion of grafted polymer-functionalized graphene sheets in the polymer matrix might contribute to the increase in thermal conductivity of the polymer nanocomposites.

\section{Conclusions}

Herein, we explored a general and convenient methodology for preparing grafted PMMA- or PS-functionalized rGO via the combination of PDA chemistry and surface-initiated ARGET-ATRP. Subsequently, the prepared PMMA- or PSmodified rGO had been used as a nanofiller in the polymer matrix. The introduction of polymer brush-decorated rGO in the PMMA or PS matrix could obviously improve the glass transition temperature $\left(T_{\mathrm{g}}\right)$, thermal stability, and thermal conductivity of the polymer matrix. The increment of $T_{\mathrm{g}}$ for PMMA/rGO-g-PMMA and PS/rGO-g-PS nanocomposites was about $15^{\circ} \mathrm{C}$ and $13^{\circ} \mathrm{C}$ higher than that for PMMA and PS, respectively. The temperature corresponding to $10 \%$ mass loss of PMMA/rGO-g-PMMA nanocomposites was enhanced to $344.8^{\circ} \mathrm{C}$. Additionally, thermal conductivity of PMMA nanocomposites was $66 \%$ higher than that of PMMA at $25^{\circ} \mathrm{C}$. This work provides a facile route to prepare polymer brush-functionalized $\mathrm{rGO}$, which could enhance the compatibility with the polymer matrices and tune properties for a wide range of applications. This strategy can also be applied to surface modification of other solid substrates.

\section{Data Availability}

The authors confirm that the data supporting the findings of this study are available within the article.

\section{Conflicts of Interest}

The authors declare that there is no conflict of interest regarding the publication of this paper.

\section{Acknowledgments}

This work was supported by the National Natural Science Foundation of China (No. 51603098), Nature Science Foundation of Shandong Province (ZR2019MEE018, ZR2020ME133), and Research Foundation for Doctors of Liaocheng University (No. 318051435).

\section{References}

[1] Y. Zhu, D. K. James, and J. M. Tour, "New Routes to Graphene, Graphene Oxide and Their Related Applications," Advanced Materials, vol. 24, no. 36, pp. 4924-4955, 2012.

[2] J. Wu, W. Pisula, and K. Mullen, "Graphenes as potential material for electronics," Chemical Reviews, vol. 107, no. 3, pp. 718-747, 2007.

[3] C. Chung, Y. K. Kim, D. Shin, S. R. Ryoo, B. H. Hong, and D. H. Min, "Biomedical applications of graphene and graphene oxide," Accounts of Chemical Research, vol. 46, no. 10, pp. 2211-2224, 2013.

[4] R. Zou, F. Liu, N. Hu et al., "Ultratough reduced graphene oxide composite films synergistically toughened and reinforced by polydopamine wrapped carbon nanotubes," Carbon, vol. 159, pp. 422-431, 2020.

[5] H. Kim, A. A. Abdala, and C. W. Macosko, "Graphene/polymer nanocomposites," Macromolecules, vol. 43, no. 16, pp. 6515-6530, 2010.

[6] K. Hu, D. D. Kulkarni, I. Choi, and V. V. Tsukruk, "Graphenepolymer nanocomposites for structural and functional applications," Progress in Polymer Science, vol. 39, no. 11, pp. 1934-1972, 2014.

[7] A. Alammar, S. H. Park, I. Ibrahim et al., "Architecting neonicotinoid-scavenging nanocomposite hydrogels for environmental remediation," Applied Materials Today, vol. 21, article 100878, 2020.

[8] A. Alammar, S. H. Park, C. J. Williams, B. Derby, and G. Szekely, "Oil-in-water separation with graphene-based nanocomposite membranes for produced water treatment," Journal of Membrane Science, vol. 603, article 118007, 2020.

[9] S. Guo and S. Dong, "Graphene nanosheet: synthesis, molecular engineering, thin film, hybrids, and energy and analytical applications," Chemical Society Reviews, vol. 40, no. 5, p. 2644, 2011.

[10] Y. Yang, J. Wang, J. Zhang, J. Liu, X. Yang, and H. Zhao, "Exfoliated graphite oxide decorated by PDMAEMA chains and polymer particles," Langmuir, vol. 25, no. 19, pp. 1180811814, 2009. 
[11] A. Nikolakopoulou, D. Tasis, L. Sygellou, V. Dracopoulos, C. Galiotis, and P. Lianos, "Study of the thermal reduction of graphene oxide and of its application as electrocatalyst in quasi-solid state dye-sensitized solar cells in combination with PEDOT," Electrochimica Acta, vol. 111, pp. 698-706, 2013.

[12] M. Moazzami Gudarzi and F. Sharif, "Enhancement of dispersion and bonding of graphene-polymer through wet transfer of functionalized graphene oxide," Express Polymer Letters, vol. 6, no. 12, pp. 1017-1031, 2012.

[13] O. C. Compton, S. W. Cranford, K. W. Putz et al., "Tuning the mechanical properties of graphene oxide paper and its associated polymer nanocomposites by controlling cooperative intersheet hydrogen bonding," ACS Nano, vol. 6, no. 3, pp. 2008-2019, 2012.

[14] S. Stankovch, R. D. Piner, X. Q. Chen, N. Q. Wu, S. T. Nguyen, and R. S. Ruoff, "Stable aqueous dispersions of graphitic nanoplatelets via the reduction of exfoliated graphite oxide in the presence of poly(sodium 4-styrenesulfonate)," Journal of Materials Chemistry, vol. 16, no. 2, pp. 155-158, 2006.

[15] X. Zhong, J. Jin, S. Li et al., "Aryne cycloaddition: highly efficient chemical modification of graphene," Chemical Communications, vol. 46, no. 39, pp. 7340-7342, 2010.

[16] J. Q. Liu, L. Tao, W. R. Yang et al., "Synthesis, characterization, and multilayer assembly of $\mathrm{pH}$ sensitive graphene-polymer nanocomposites," Langmuir, vol. 26, no. 12, pp. 1006810075, 2010.

[17] M. K. Sung, S. Park, D. Kim, S. Y. Park, R. S. Ruoff, and H. Lee, "Simultaneous Reduction and Surface Functionalization of Graphene Oxide by Mussel-Inspired Chemistry," Advanced Functional Materials, vol. 21, no. 1, pp. 108-112, 2011.

[18] X. Hu, R. Qi, J. Zhu et al., "Preparation and properties of dopamine reduced graphene oxide and its composites of epoxy," Journal of Applied Polymer Science, vol. 131, no. 2, article 39754, 2014.

[19] L. Q. Xu, W. J. Yang, K. G. Neoh, E. T. Kang, and G. D. Fu, "Dopamine-induced reduction and functionalization of graphene oxide nanosheets," Macromolecules, vol. 43, no. 20, pp. 8336-8339, 2010.

[20] Y. Song, G. Ye, F. Wu et al., "Bioinspired polydopamine (PDA) chemistry meets ordered mesoporous carbons (OMCs): a benign surface modification strategy for versatile functionalization," Chemistry of Materials, vol. 28, no. 14, pp. 50135021, 2016.

[21] Z. Jia, H. Li, Y. Zhao et al., "Electrical and mechanical properties of poly(dopamine)-modified copper/reduced graphene oxide composites," Journal of Materials Science, vol. 52, no. 19, pp. 11620-11629, 2017.

[22] J. Liu, Y. Ye, Y. Xue, X. Xie, and Y. W. Mai, "Recent advances in covalent functionalization of carbon nanomaterials with polymers: Strategies and perspectives," Journal of Polymer Science, Part A: Polymer Chemistry, vol. 55, no. 4, pp. 622-631, 2017.

[23] R. K. Layek and A. K. Nandi, "A review on synthesis and properties of polymer functionalized graphene," Polymer, vol. 54, no. 19, pp. 5087-5103, 2013.

[24] V. D. Punetha, S. Rana, H. J. Yoo et al., "Functionalization of carbon nanomaterials for advanced polymer nanocomposites: a comparison study between CNT and graphene," Progress in Polymer Science, vol. 67, pp. 1-47, 2017.

[25] P. Gao, M. Liu, J. Tian et al., "Improving the drug delivery characteristics of graphene oxide based polymer nanocomposites through the "one-pot" synthetic approach of single- electron-transfer living radical polymerization," Applied Surface Science, vol. 378, pp. 22-29, 2016.

[26] J. O. Zoppe, N. C. Ataman, P. Mocny, J. Wang, J. Moraes, and H. A. Klok, "Surface-initiated controlled radical polymerization: state-of-the-art, opportunities, and challenges in surface and interface engineering with polymer brushes," Chemical Reviews, vol. 117, no. 3, pp. 1105-1318, 2017.

[27] Z. Hu, Q. Shao, M. G. Moloney et al., "Nondestructive functionalization of graphene by surface-initiated atom transfer radical polymerization: an ideal nanofiller for poly(p-phenylene benzobisoxazole) fibers," Macromolecules, vol. 50, no. 4, pp. 1422-1429, 2017.

[28] H. Roghani-Mamaqani and K. Khezri, "A grafting from approach to graft polystyrene chains at the surface of graphene nanolayers by RAFT polymerization: various graft densities from hydroxyl groups," Applied Surface Science, vol. 360, pp. 373-382, 2016.

[29] Y. Yang, X. Song, L. Yuan et al., "Synthesis of PNIPAM polymer brushes on reduced graphene oxide based on click chemistry and RAFT polymerization," Journal of Polymer Science Part A: Polymer Chemistry, vol. 50, no. 2, pp. 329-337, 2012.

[30] Y. Song, J. Lu, B. Liu, and C. Lu, “Temperature responsive polymer brushes grafted from graphene oxide: an efficient fluorescent sensing platform for 2,4,6-trinitrophenol," Journal of Materials Chemistry C, vol. 4, no. 29, pp. 7083-7092, 2016.

[31] A. Badri, M. R. Whittaker, and P. B. Zetterlund, "Modification of graphene/graphene oxide with polymer brushes using controlled/living radical polymerization," Journal of Polymer Science Part A: Polymer Chemistry, vol. 50, no. 15, pp. 29812992, 2012.

[32] H. Au, N. Rubio, and M. S. P. Shaffer, "Brominated graphene as a versatile precursor for multifunctional grafting," Chemical Science, vol. 9, no. 1, pp. 209-217, 2018.

[33] S. Liu, Y. Zhu, Y. Li et al., "Preparation of graphene/poly(2hydroxyethyl acrylate) nanohybrid materials via an ambient temperature "grafting-from" strategy," Polymer Chemistry, vol. 6, no. 2, pp. 311-321, 2015.

[34] P. Ding, J. Zhang, N. Song, S. Tang, Y. Liu, and L. Shi, “Growing polystyrene chains from the surface of graphene layers via RAFT polymerization and the influence on their thermal properties," Composites Part A: Applied Science and Manufacturing, vol. 69, pp. 186-194, 2015.

[35] P. Eskandari, Z. Abousalman-Rezvani, H. Roghani-Mamaqani, M. Salami-Kalajahi, and H. Mardani, "Polymer grafting on graphene layers by controlled radical polymerization," Advances in Colloid and Interface Science, vol. 273, article 102021, 2019.

[36] O. García-Valdez, R. Ledezma-Rodríguez, E. Saldívar-Guerra, L. Yate, S. Moya, and R. F. Ziolo, "Graphene oxide modification with graft polymers via nitroxide mediated radical polymerization," Polymer, vol. 55, no. 10, pp. 2347-2355, 2014.

[37] S. Railian, J. J. Haven, L. Maes et al., "Photo-induced coppermediated (meth)acrylate polymerization towards graphene oxide and reduced graphene oxide modification," European Polymer Journal, vol. 134, article 109810, 2020.

[38] Z. Zhang, P. Zhang, Y. Wang, and W. Zhang, "Recent advances in organic-inorganic well-defined hybrid polymers using controlled living radical polymerization techniques," Polymer Chemistry, vol. 7, no. 24, pp. 3950-3976, 2016.

[39] A. Zeliha, A. Fabrice, H. Amy, O. C. Brendan, and H. Andreas, "Functional Brush-Decorated Poly(globalide) Films by 
ARGET-ATRP for Bioconjugation," Macromolecular Bioscience, vol. 14, article 1600, 2014.

[40] B. Wang, Z. Wang, F. Jiang, H. Fang, and Z. Wang, "Synthesis and characterization of MWCNT-graft-polyisoprene via ARGET ATRP," RSC Advances, vol. 4, no. 50, article 26468, 2014.

[41] Y. Song, G. Ye, Y. Lu, J. Chen, J. Wang, and K. Matyjaszewski, "Surface-initiated ARGET ATRP of poly(glycidyl methacrylate) from carbon nanotubes via bioinspired catechol chemistry for efficient adsorption of uranium ions," ACS Macro Letters, vol. 5, no. 3, pp. 382-386, 2016.

[42] T. B. Mai, T. N. Tran, M. Rafiqul Islam, J. M. Park, and K. T. Lim, "Covalent functionalization of silica nanoparticles with poly(N-isopropylacrylamide) employing thiol-ene chemistry and activator regenerated by electron transfer ATRP protocol," Journal of Materials Science, vol. 49, no. 4, pp. 15191526, 2014.

[43] C. N. Yan, L. Xu, Q. D. Liu et al., "Surface-induced ARGET ATRP for silicon nanoparticles with fluorescent polymer brushes," Polymers, vol. 11, no. 7, article 1228, 2019.

[44] J. Sha, Y. Gao, T. Wu et al., "Biocompatible graphene nanosheets grafted with poly(2-hydroxyethyl methacrylate) brushes via surface-initiated ARGET ATRP," RSC Advances, vol. 6, no. 42, pp. 35641-35647, 2016.

[45] S. Wang, H. Meng, Y. Li et al., "Polymer brushes grafted from graphene via bioinspired polydopamine chemistry and activators regenerated by electron transfer atom transfer radical polymerization," Journal of Polymer Science Part A: Polymer Chemistry, vol. 57, no. 6, pp. 689-698, 2019.

[46] S. Wang, J. Song, Y. Li et al., "Grafting antibacterial polymer brushes from titanium surface via polydopamine chemistry and activators regenerated by electron transfer ATRP," Reactive and Functional Polymers, vol. 140, pp. 48-55, 2019.

[47] J. M. Bak, T. Lee, E. Seo et al., "Thermoresponsive graphene nanosheets by functionalization with polymer brushes," Polymer, vol. 53, no. 2, pp. 316-323, 2012.

[48] J. M. Bak and H. Lee, "pH-tunable aqueous dispersion of graphene nanocomposites functionalized with poly(acrylic acid) brushes," Polymer, vol. 53, no. 22, pp. 4955-4960, 2012.

[49] L. Zhu, S. Su, and J. M. Hossenlopp, "Thermal stability and fire retardancy of PMMA (nano)composites with layered metal hydroxides containing dodecyl sulfate anions," Polymers for Advanced Technologies, vol. 23, no. 2, pp. 171-181, 2012.

[50] X. Wang, Q. Su, Y. Hu, C. Wang, and J. Zheng, "Structure and thermal stability of PMMA/MMT nanocomposites as denture base material," Journal of Thermal Analysis and Calorimetry, vol. 115, no. 2, pp. 1143-1151, 2014.

[51] J. Du and H. M. Cheng, "The fabrication, properties, and uses of graphene/polymer composites," Macromolecular Chemistry and Physics, vol. 213, no. 10-11, pp. 1060-1077, 2012.

[52] J. R. Potts, D. R. Dreyer, C. W. Bielawski, and R. S. Ruoff, "Graphene-based polymer nanocomposites," Polymer, vol. 52, no. 1, pp. 5-25, 2011. 